\title{
Diffuse myocardial fibrosis in hypertrophic cardiomyopathy can be identified by cardiovascular magnetic resonance, and is associated with left ventricular diastolic dysfunction
}

Andris H Ellims ${ }^{1,2}$, Leah M lles ${ }^{1,2}$, Liang-han Ling ${ }^{1,2}$, James L Hare ${ }^{1,2}$, David M Kaye ${ }^{1,2}$ and Andrew J Taylor $r^{1,2^{*}}$

\begin{abstract}
Background: The presence of myocardial fibrosis is associated with worse clinical outcomes in hypertrophic cardiomyopathy (HCM). Cardiovascular magnetic resonance (CMR) with late gadolinium enhancement (LGE) sequences can detect regional, but not diffuse myocardial fibrosis. Post-contrast $T_{1}$ mapping is an emerging CMR technique that may enable the non-invasive evaluation of diffuse myocardial fibrosis in HCM. The purpose of this study was to non-invasively detect and quantify diffuse myocardial fibrosis in HCM with CMR and examine its relationship to diastolic performance.
\end{abstract}

Methods: We performed CMR on 76 patients - 51 with asymmetric septal hypertrophy due to HCM and 25 healthy controls. Left ventricular (LV) morphology, function and distribution of regional myocardial fibrosis were evaluated with cine imaging and LGE. A CMR $T_{1}$ mapping sequence determined the post-contrast myocardial $T_{1}$ time as an index of diffuse myocardial fibrosis. Diastolic function was assessed by transthoracic echocardiography.

Results: Regional myocardial fibrosis was observed in $84 \%$ of the HCM group. Post-contrast myocardial $\mathrm{T}_{1}$ time was significantly shorter in patients with HCM compared to controls, consistent with diffuse myocardial fibrosis (498 \pm 80 ms vs. $561 \pm 47$ ms, $p<0.001$ ). In HCM patients, post-contrast myocardial $T_{1}$ time correlated with mean E/e' $(r=-0.48, p<0.001)$.

Conclusions: Patients with HCM have shorter post-contrast myocardial $T_{1}$ times, consistent with diffuse myocardial fibrosis, which correlate with estimated LV filling pressure, suggesting a mechanistic link between diffuse myocardial fibrosis and abnormal diastolic function in HCM.

Keywords: Hypertrophic cardiomyopathy, Magnetic resonance imaging, Myocardial fibrosis, $T_{1}$ mapping

\section{Background}

Hypertrophic cardiomyopathy $(\mathrm{HCM})$ is a relatively common inherited cardiac disease defined by the presence of otherwise unexplained left ventricular (LV) hypertrophy associated with non-dilated ventricular chambers [1,2]. Inheritance is autosomal dominant and

\footnotetext{
* Correspondence: Andrew.Taylor@bakeridi.edu.au

${ }^{1}$ Heart Centre, Alfred Hospital, Melbourne, Australia

${ }^{2}$ Baker IDI Heart and Diabetes Research Institute Melbourne, Australia
}

mutations generally involve sarcomeric genes [3,4]. Patients may develop symptoms from LV outflow tract (LVOT) obstruction [2] and, in the presence of certain high-risk features, an implantable cardioverter defibrillator is recommended to reduce the risk of sudden cardiac death [5]. Many patients, however, develop symptoms of breathlessness due to diastolic dysfunction which is largely independent of the severity of LVOT obstruction [6]. As patients with HCM are known to develop diffuse, as well as regional myocardial fibrosis, this may 
represent a mechanism for increased myocardial stiffness leading to impaired diastolic filling [7].

Cardiovascular magnetic resonance (CMR) after intravenous administration of gadolinium contrast can noninvasively characterize myocardial tissue [8]. Late gadolinium enhancement (LGE) in HCM patients represents replacement fibrosis histologically $[9,10]$ and portends a worse outcome [11]. LGE has been noted in up to $80 \%$ of HCM patients [12-14], typically within the thickened interventricular septum or at points of insertion of the RV free wall $[15,16]$. Histologic evaluation of myocardial tissue in HCM, however, has demonstrated a more global, or diffuse, increase in fibrosis that cannot be detected by standard CMR LGE sequences $[7,10]$.

Post-contrast myocardial longitudinal relaxation time $\left(\mathrm{T}_{1}\right)$ mapping is an emerging CMR technique that can detect and quantify diffuse interstitial myocardial fibrosis [17] without the necessity for invasive biopsy. Different $\mathrm{T}_{1}$ mapping protocols have identified diffuse myocardial fibrosis in several cardiac disease states $[8,18]$, however research involving $\mathrm{HCM}$ is limited $[19,20]$.

A comprehensive non-invasive evaluation of regional and diffuse myocardial fibrosis in a typical cohort of HCM patients has not previously been described. This study was undertaken to detect and quantify diffuse myocardial fibrosis in these patients using a histologicallyvalidated CMR post-contrast myocardial $\mathrm{T}_{1}$ mapping technique [17]. Furthermore, we investigated the relationships of both patterns of fibrosis to LV diastolic performance and clinical manifestations.

\section{Methods}

\section{Patient selection}

All research was performed at the Alfred Hospital, Melbourne, Australia between August 2010 and October 2011. Fifty-one consecutive patients (39 men, 12 women) referred to our CMR department for the further evaluation of asymmetric septal hypertrophy (ASH) due to HCM were invited to participate. ASH was defined as an interventricular septum thickness of $\geq 15 \mathrm{~mm}$ with a ratio of septal-to-lateral ventricular wall thickness of $\geq 1.3: 1.0$ as measured by echocardiography, and the diagnosis of HCM required the absence of another condition that could cause the degree of hypertrophy observed [1]. Twenty-five asymptomatic subjects with no documented history of cardiovascular disease formed a healthy control group.

Exclusion criteria included previous septal reduction therapy; previously documented coronary artery disease or current symptoms suggestive of coronary artery disease; atrial fibrillation; diabetes mellitus; contraindications to CMR, including pacemaker and defibrillator implantation; and significant renal dysfunction (estimated glomerular filtration rate $(\mathrm{eGFR})<30 \mathrm{~mL} / \mathrm{min} / 1.73 \mathrm{~m}^{2}$ ).
Informed consent was obtained from all participants and the study was conducted in accordance with the Alfred Hospital Ethics Committee's guidelines.

\section{CMR protocol \\ CMR sequences}

We performed CMR using a clinical 1.5-T scanner (Signa HD 1.5-T, GE Healthcare, Waukesha, Wisconsin, USA). All sequences were acquired during a breath-hold of 10-15 s. LV function was assessed by a steady-state free precession (SSFP) pulse sequence (repetition time $[\mathrm{TR}]=3.8 \mathrm{~ms}$, echo time $[\mathrm{TE}]=1.6 \mathrm{~ms}, 30$ phases, slice thickness $8 \mathrm{~mm}$ ).

Initial cine CMR sequences were performed in 3 standard long-axis (4-, 3- and 2-chamber views) and short-axis slices (basal, mid, and apical), kept identical for each subsequent sequence throughout the CMR examination [21]. From an end-diastolic, 4-chamber, long-axis view, 5 equally spaced short-axis slices were planned, so that the 2 outer slices lined up exactly either with the tip of the apex or the mitral annulus. The 2 outer slices were then deleted, leaving 3 slices corresponding to typical basal, mid, and apical short-axis views. To calculate LV volume and function, a contiguous short-axis SSFP stack was acquired $(8 \mathrm{~mm}$ slice thickness, no gap), extending from the mitral valve annulus to the LV apex.

LGE was evaluated 10 min after a bolus of gadolinium-diethylene triamine penta-acetic acid (DTPA) (0.2 mmol/kg BW Magnevist, Schering, Germany) to identify regional fibrosis using a $\mathrm{T}_{1}$-weighted inversion recovery gradient echo technique (TR 7.1 ms, TE $3.1 \mathrm{~ms}$, inversion time [TI] individually determined to null the myocardial signal, slice thickness $8 \mathrm{~mm}$, matrix $256 \times 192$, number of acquisitions $=2$ ). The TI optimization sequence was performed 8 min post-gadolinium administration and was a fast gradient echo, inversion recovery, gated, multi-phase acquisition, commencing at an inversion time of $150 \mathrm{~ms}$ and increasing in $25 \mathrm{~ms}$ increments to $250 \mathrm{~ms}$, in a single mid-ventricular short-axis slice. A visual determination of the optimum TI to null the myocardial signal was then made. LGE imaging was performed using both standard long-axis and short-axis views of the LV (including a contiguous stack of slices from the mitral valve annulus to the apex).

For the evaluation of diffuse myocardial fibrosis, a $\mathrm{T}_{1}$ mapping sequence was used to cycle through acquisition of images obtained at the 3 standard short-axis levels over a range of inversion times [17]. The sequence consisted of an electrocardiogram-triggered, inversionrecovery prepared, 2-dimensional fast gradient echo sequence employing variable temporal sampling of -space (VAST) [22] (Global Applied Science Laboratory, GE Healthcare). Ten images were acquired sequentially at 
increasing inversion times (75 to $750 \mathrm{~ms}$ ), commencing $20 \mathrm{~min}$ after the bolus of gadolinium-DTPA and over a series of 3 to 5 breath-holds. Imaging parameters were TR/TE: $3.7 \mathrm{~ms} / 1.2 \mathrm{~ms}$, flip angle: $20^{\circ}, 256 \times 128$ acquisition matrix, $36 \times 27 \mathrm{~cm}$ field of view, slice thickness 8 $\mathrm{mm}$, TI: 75-750 ms, trigger delay $300 \mathrm{~ms}$, and views per segment $=24$. The acquisition of all ten images for each myocardial slice was completed in approximately three minutes. These images were then processed with a curve fitting technique to generate $\mathrm{T}_{1}$ maps.

\section{Evaluation of LV dimensions, function, mass and regional fibrosis}

Volumetric analysis of the LV was performed using the summation of disc method. Regional fibrosis was identified by LGE within the myocardium, defined quantitatively by a myocardial post-contrast signal intensity $6 \mathrm{SD}$ above that within a reference region of remote myocardium (without LGE) within the same slice [23]. LGE was defined as being present only if it was identified in two orthogonal views.

\section{Evaluation of diffuse fibrosis with $T_{1}$ mapping}

Following image acquisition, the ten short-axis images of varying inversion times were transferred to an external computer for analysis using a dedicated research software package (Cinetool, Global Applied Science Laboratory, GE Healthcare). This provided the ability to analyze regions of interest (ROIs) to find average $T_{1}$ for that area, as well as a pixel-by-pixel determination of $\mathrm{T}_{1}$, by fitting data acquired at various preparation times to the exponential curve: $\mathrm{M}_{\mathrm{z}}(\mathrm{t}=\mathrm{TI})=\mathrm{M}_{0}\left(\mathrm{~A}-\mathrm{B}\left[\mathrm{e}^{-\mathrm{t} / \mathrm{T} 1}=\right]\right)$, relating the sample magnetization $M_{z}$ observed at the time $t=T I$ to the equilibrium magnetization $M_{0}$ and sample $\mathrm{T}_{1}$, where TI denotes inversion time for an inversion recovery experiment. For each short-axis image, a ROI was drawn around the entire LV myocardium (excluding papillary muscles) to calculate post-contrast myocardial $\mathrm{T}_{1}$ time. In subjects with regional fibrosis detected by LGE, these regions were excluded from the ROI for the primary analysis of post-contrast myocardial $\mathrm{T}_{1}$ time (see Figure 1). To investigate for regional variations in $\mathrm{T}_{1}$ time, separate ROIs were drawn around hypertrophied (defined as CMR-measured wall thickness $\geq 11 \mathrm{~mm}$ during diastole) and non-hypertrophied LV myocardium for each short-axis slice.

\section{Evaluation of diastolic function}

Transthoracic echocardiography with a standard clinical protocol was performed on all patients immediately prior to CMR. Diastolic function was assessed by a combination of mitral inflow pattern (E to A ratio and deceleration time) and mitral annular velocities (e', measured at the septal and lateral aspects of the mitral annulus in the apical 4-chamber view). Additionally, mitral E/e' (septal, lateral and mean) was chosen as an index of LV filling pressure.

\section{Image analysis}

All CMR and echocardiogram images were interpreted by two experienced readers unaware of the subjects' clinical information and the results of other diagnostic tests. Endocardial and epicardial LV contours were drawn manually for each diastolic and systolic frame, excluding papillary muscles.

\section{Statistical methods}

All data are expressed as mean \pm SD unless otherwise indicated. Comparison of continuous variables utilized unpaired Student t-test. Comparisons of proportions were made with chi-squared analysis. Multiple comparisons were performed by analysis of variance (ANOVA), with post hoc testing (Holm-Sidak method) as appropriate. Correlations of variables were determined by calculating the Pearson Product Moment. Multiple linear regression was used to determine the independence of
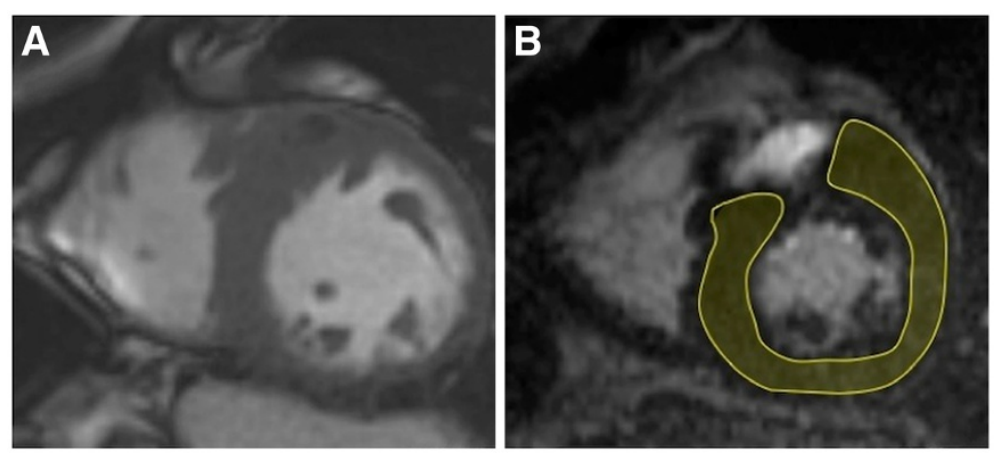

Figure 1 Calculation of post-contrast myocardial $\mathbf{T}_{\mathbf{1}}$ time in HCM. (A) Short-axis steady-state free precession image of a patient with asymmetric septal hypertrophy due to HCM. (B) Post-contrast $\mathrm{T}_{1}$ mapping image at the same short-axis level, with the region of interest (shaded) chosen to include LV myocardium, but exclude a region of late gadolinium enhancement. 
correlations observed on simple linear regression, with all correlations with a $\mathrm{p}$ value $<0.1$ entered into multiple linear regression analysis. Binary categorical variables were entered into the analyses using dummy coding. Linear regression and Bland-Altman analysis were performed to assess interobserver agreement. For all comparisons, a $\mathrm{p}$ value of $<0.05$ was considered significant, and all reported $\mathrm{p}$ values are 2-tailed. All analyses were conducted using Stata software version 11.1 (StataCorp, College Station, Texas).

\section{Results}

\section{Clinical and demographic data}

A total of 76 patients were evaluated during the study period, comprising 51 patients with ASH due to HCM and 25 control subjects. Baseline characteristics of both groups are presented in Table 1. Patients in both groups were of a similar age. $76 \%$ of HCM patients were male, compared to $72 \%$ of control subjects. Body mass index (BMI) was significantly higher in the HCM group (27.5 $\pm 4.9 \mathrm{~kg} / \mathrm{m}^{2}$ vs. $\left.24.1 \pm 2.7 \mathrm{~kg} / \mathrm{m}^{2}, \mathrm{p}<0.001\right)$. One-third of HCM patients had a first-degree relative previously diagnosed with HCM. 74\% of patients reported symptoms attributable to HCM (including chest pain, dyspnoea, presyncope and/or syncope). The severity of dyspnoea in HCM patients was generally mild, with no patients experiencing New York Heart Association

\section{Table 1 Baseline characteristics}

\begin{tabular}{|c|c|c|c|}
\hline & $\mathrm{HCM}(n=51)$ & Control $(n=25)$ & $p$ value \\
\hline Age, y & $48 \pm 14$ & $48 \pm 17$ & 0.8 \\
\hline Males, n (\%) & $39(76 \%)$ & $18(72 \%)$ & 0.7 \\
\hline Body mass index, $\mathrm{kg} / \mathrm{m}^{2}$ & $27.5 \pm 4.9$ & $24.1 \pm 2.7$ & $<0.001$ \\
\hline $\begin{array}{l}\text { Family history of } \mathrm{HCM} \text {, } \\
\mathrm{n}(\%)\end{array}$ & $17(33 \%)$ & - & \\
\hline \multicolumn{4}{|l|}{ Symptoms, n (\%) } \\
\hline Chest pain & 16 (31\%) & - & \\
\hline Dyspnoea & $28(55 \%)$ & - & \\
\hline NYHA class I or II & $51(100 \%)$ & - & \\
\hline Presyncope & 18 (35\%) & - & \\
\hline Syncope & $6(12 \%)$ & - & \\
\hline \multicolumn{4}{|l|}{ Medications, n (\%) } \\
\hline Beta-blocker & $29(57 \%)$ & - & \\
\hline Calcium channel blocker & $10(20 \%)$ & - & \\
\hline $\begin{array}{l}\text { Resting heart rate, } \\
\text { beats } / \mathrm{min}\end{array}$ & $60 \pm 10$ & $62 \pm 9$ & 0.6 \\
\hline $\begin{array}{l}\text { Systolic blood pressure, } \\
\mathrm{mm} \mathrm{Hg}\end{array}$ & $129 \pm 16$ & $133 \pm 18$ & 0.4 \\
\hline Hematocrit & $0.43 \pm 0.04$ & $0.42 \pm 0.03$ & 0.3 \\
\hline $\mathrm{eGFR}, \mathrm{ml} / \mathrm{min} / 1.73 \mathrm{~m}^{2}$ & $81 \pm 12$ & $86 \pm 8$ & 0.14 \\
\hline
\end{tabular}

NYHA indicates New York Heart Association; and eGFR, estimated glomerular filtration rate.
(NYHA) class III/IV symptoms. Three-quarters of the HCM group were receiving beta-blocker and/or nondihydropyridine calcium channel blocker therapy. There were no significant differences in heart rate, systolic blood pressure, hematocrit, or renal function between the HCM and control groups.

\section{CMR findings}

CMR was successfully completed in all 76 patients and the results are displayed in Table 2. The HCM group had a significantly higher LV ejection fraction and a greater LV mass indexed to body surface area (BSA) compared to the control group. LV end-diastolic volumes indexed to BSA were similar in both groups. The maximum ventricular septal thickness of HCM patients was $20 \pm 3 \mathrm{~mm}$ compared to $8 \pm 2 \mathrm{~mm}$ for control subjects, while the ratio of septal-to-lateral ventricular wall thickness for the HCM group was 2.3:1. LGE was observed in $84 \%$ of HCM patients, generally localized to the ventricular septum or points of RV free wall insertion. Subendocardially-based LGE, consistent with ischemic scar, was not observed in any patient. The mean quantity of LGE, expressed as a percentage of LV mass, was $6.1 \pm 7.7 \%$.

\section{Post-contrast myocardial $\mathrm{T}_{1}$ time in $\mathrm{HCM}$ and control subject}

The timing of acquisition of $T_{1}$ mapping sequences after the delivery of the gadolinium contrast bolus was similar in both groups $(23: 43 \pm 3: 57 \mathrm{~min}$ vs. $22: 34 \pm 3: 48 \mathrm{~min}$, $\mathrm{p}=0.2$ ). There was an excellent correlation between the two blinded CMR specialist reviewers when they independently calculated myocardial $\mathrm{T}_{1}$ times $(\mathrm{r}=0.99$, $\mathrm{p}<0.001$ ). Bland-Altman analysis showed good inter-

Table 2 Cardiac MRI data

\begin{tabular}{llll}
\hline & HCM (n=51) & Control (n=25) & p value \\
\hline LVEDV, ml & $162 \pm 36$ & $156 \pm 35$ & 0.5 \\
LVEDV indexed, ml/BSA & $81 \pm 14$ & $83 \pm 13$ & 0.5 \\
LVESV, ml & $50 \pm 17$ & $63 \pm 18$ & $<0.01$ \\
LV stroke volume, ml & $112 \pm 27$ & $94 \pm 20$ & $<0.01$ \\
LVEF, \% & $70 \pm 7$ & $60 \pm 6$ & $<0.001$ \\
LV mass, g & $178 \pm 54$ & $98 \pm 25$ & $<0.001$ \\
LV mass indexed, g/BSA & $89 \pm 25$ & $52 \pm 9$ & $<0.001$ \\
Septal thickness, mm & $20 \pm 3$ & $8 \pm 2$ & $<0.001$ \\
Lateral wall thickness, mm & $9 \pm 2$ & $8 \pm 1$ & $<0.05$ \\
Septal:lateral wall thickness & $2.3 \pm 0.7$ & $1.1 \pm 0.9$ & $<0.001$ \\
Presence of LGE, n (\%) & $43(84 \%)$ & $0(0 \%)$ & \\
Quantity of LGE, \% of & $6.1 \pm 7.7$ & 0 & \\
LV mass & & & \\
\hline
\end{tabular}

LVEDV indicates left ventricular end-diastolic volume; LVEF, left ventricular ejection fraction; LVESV, left ventricular end-systolic volume; and LGE, late gadolinium enhancement. 
observer agreement (mean difference in $\mathrm{T}_{1}$ time was $0.29 \pm 19.28 \mathrm{~ms}$, limits of agreement were -38.29 to 38.84). Patients with HCM had significantly shorter post-contrast myocardial $\mathrm{T}_{1}$ times compared with controls $(498 \pm 80 \mathrm{~ms}$ vs. $561 \pm 47 \mathrm{~ms}, \mathrm{p}<0.0001)$ (Table 3$)$. When regions of LGE were included in the analysis of HCM patients, a further reduction in $\mathrm{T}_{1}$ time $(483 \pm 85$ ms) was observed. Post-contrast $T_{1}$ times were similar in both hypertrophied and non-hypertrophied LV myocardium (503 $\pm 127 \mathrm{~ms}$ vs. $497 \pm 111 \mathrm{~ms}, \mathrm{p}=0.7)$. There was no difference in post-contrast $\mathrm{T}_{1}$ times of the LV blood pool between the HCM and control groups (304 $\pm 31 \mathrm{~ms}$ vs. $306 \pm 22 \mathrm{~ms}$ respectively, $\mathrm{p}=0.8$ ).

\section{Echocardiography findings}

Echocardiographic data are presented in Table 4. Left atrial volume indexed to BSA was higher in the HCM group. The mean resting LVOT gradient in HCM patients was $26 \pm 36 \mathrm{~mm} \mathrm{Hg}$. Septal, lateral, and mean early diastolic mitral annular velocities (e') as measured by tissue Doppler imaging (TDI) were all lower in the HCM group compared to controls, and septal, lateral, and mean E/e' were higher in the HCM group.

\section{Correlates of post-contrast myocardial $\mathrm{T}_{1}$ time in $\mathrm{HCM}$ patients}

Significant negative correlations were observed between post-contrast myocardial $\mathrm{T}_{1}$ time and age and BMI (Table 5). Following multiple linear regression analysis, the correlation between $\mathrm{T}_{1}$ time and age remained significant. There were no associations between $\mathrm{T}_{1}$ time and the presence of symptoms or NYHA class. There were no significant correlations between $\mathrm{T}_{1}$ time and resting heart rate, blood pressure, hematocrit or eGFR. The presence and quantity of LGE (expressed as a percentage of LV mass) did not correlate with post-contrast myocardial $\mathrm{T}_{1}$ time.

\section{Correlates of diastolic dysfunction in HCM patients}

Using mean E/e' as a measure of diastolic dysfunction, simple linear regression demonstrated significant positive correlations with age, indexed LV mass, maximum septal thickness, indexed left atrial volume, and resting

Table 3 Post-contrast myocardial $T_{1}$ mapping data

\begin{tabular}{|c|c|c|c|}
\hline & $\operatorname{HCM}(n=51)$ & Control $(n=25)$ & $p$ value \\
\hline \multicolumn{4}{|l|}{ Post-contrast $\mathrm{T}_{1}$ time, ms } \\
\hline LV myocardium, excluding LGE & $498 \pm 80$ & $561 \pm 47$ & $<0.0001$ \\
\hline LV myocardium, including LGE & $483 \pm 85$ & $561 \pm 47$ & $<0.0001$ \\
\hline LV blood pool & $304 \pm 31$ & $306 \pm 22$ & 0.8 \\
\hline
\end{tabular}

LGE indicates late gadolinium enhancement.
Table 4 Echocardiography data

\begin{tabular}{llll}
\hline & HCM $(\mathbf{n}=\mathbf{5 1})$ & Control $(\mathbf{n}=\mathbf{2 5})$ & p value \\
\hline $\begin{array}{l}\text { Left atrial volume indexed, } \\
\mathrm{ml} / \mathrm{m}^{2}\end{array}$ & $51 \pm 18$ & $32 \pm 10$ & $<0.001$ \\
Resting LVOT gradient, & $26 \pm 36$ & $4 \pm 1$ & $<0.001$ \\
$\mathrm{~mm} \mathrm{Hg}$ & & & \\
Mitral E velocity, cm/s & $0.8 \pm 0.2$ & $0.7 \pm 0.2$ & 0.1 \\
Mitral A velocity, cm/s & $0.6 \pm 0.3$ & $0.6 \pm 0.2$ & 0.5 \\
E/A ratio & $1.4 \pm 0.6$ & $1.4 \pm 0.5$ & 0.7 \\
Deceleration time, ms & $217 \pm 51$ & $188 \pm 36$ & $<0.01$ \\
Septal e', cm/s & $6.0 \pm 1.7$ & $8.8 \pm 3.0$ & $<0.001$ \\
Lateral e', cm/s & $8.3 \pm 2.6$ & $12.0 \pm 4.0$ & $<0.001$ \\
Mean e', cm/s & $7.1 \pm 1.9$ & $10.4 \pm 3.3$ & $<0.001$ \\
Septal E/e' ratio & $14.4 \pm 5.8$ & $8.9 \pm 3.2$ & $<0.001$ \\
Lateral E/e' ratio & $10.6 \pm 4.7$ & $6.4 \pm 1.9$ & $<0.001$ \\
Mean E/e' ratio & $12.5 \pm 4.9$ & $7.7 \pm 2.4$ & $<0.001$ \\
\hline
\end{tabular}

LVOT indicates left ventricular outflow tract.

LVOT gradient. A negative correlation was observed between mean $\mathrm{E} / \mathrm{e}^{\prime}$ and post-contrast myocardial $\mathrm{T}_{1}$ time (see Figure 2). No significant correlation was observed between the amount of LGE and mean E/e'. In multivariate analysis, the correlation between mean $\mathrm{E} / \mathrm{e}^{\prime}$ and $\mathrm{T}_{1}$ time remained significant (Table 6).

Table 5 Predictors of post-contrast myocardial $T_{1}$ time in HCM group by simple and multiple linear regression

\begin{tabular}{|c|c|c|c|c|}
\hline & \multicolumn{2}{|c|}{ Simple linear regression } & \multicolumn{2}{|c|}{ Multiple linear regression } \\
\hline & $r$ & $p$ value & $\beta$ & p value \\
\hline Age & -0.41 & $<0.01$ & -0.34 & 0.02 \\
\hline Male & -0.14 & 0.3 & & \\
\hline $\begin{array}{l}\text { Body mass } \\
\text { index }\end{array}$ & -0.35 & $<0.05$ & -0.24 & 0.08 \\
\hline $\begin{array}{l}\text { Family history } \\
\text { of HCM }\end{array}$ & 0.20 & 0.2 & & \\
\hline $\begin{array}{l}\text { Presence of } \\
\text { symptoms }\end{array}$ & -0.17 & 0.2 & & \\
\hline NYHA class & -0.16 & 0.3 & & \\
\hline $\begin{array}{l}\text { Resting heart } \\
\text { rate }\end{array}$ & 0.13 & 0.4 & & \\
\hline $\begin{array}{l}\text { Systolic blood } \\
\text { pressure }\end{array}$ & -0.12 & 0.4 & & \\
\hline $\begin{array}{l}\text { Diastolic blood } \\
\text { pressure }\end{array}$ & 0.12 & 0.4 & & \\
\hline Hematocrit & -0.29 & 0.11 & & \\
\hline eGFR & 0.26 & 0.3 & & \\
\hline $\begin{array}{l}\text { Presence of } \\
\text { LGE }\end{array}$ & 0.18 & 0.21 & & \\
\hline $\begin{array}{l}\text { Quantity of } \\
\text { LGE }\end{array}$ & -0.13 & 0.39 & & \\
\hline
\end{tabular}

NYHA indicates New York Heart Association; eGFR, estimated glomerular filtration rate; and LGE, late gadolinium enhancement. 


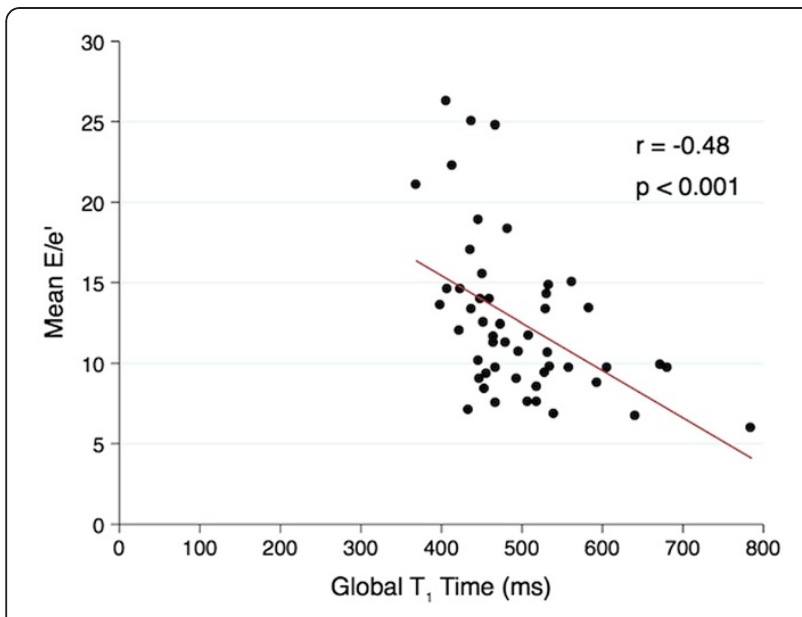

Figure 2 Post-contrast myocardial $\mathrm{T}_{1}$ time and mean $\mathrm{E} / \mathrm{e}^{\prime}$ in HCM patients. A significant negative correlation was observed between post-contrast myocardial $\mathrm{T}_{1}$ time and mean $\mathrm{E} / \mathrm{e}^{\prime}(r=-0.48$, $p<0.001)$.

Table 6 Predictors of mean E/e' in HCM group by simple and multiple line regression

\begin{tabular}{|c|c|c|c|c|}
\hline & \multicolumn{2}{|c|}{ Simple linear regression } & \multicolumn{2}{|c|}{ Multiple linear regression } \\
\hline & $r$ & $p$ value & $\beta$ & $p$ value \\
\hline \multicolumn{5}{|c|}{ Baseline characteristics } \\
\hline Age & 0.33 & $<0.05$ & 0.11 & 0.4 \\
\hline $\begin{array}{l}\text { Body mass } \\
\text { index }\end{array}$ & 0.05 & 0.7 & & \\
\hline NYHA class & 0.20 & 0.15 & & \\
\hline \multicolumn{5}{|l|}{ CMR parameters } \\
\hline LVEDV indexed & 0.23 & 0.11 & & \\
\hline LVEF & -0.10 & 0.5 & & \\
\hline $\begin{array}{l}\text { LV mass } \\
\text { indexed }\end{array}$ & 0.30 & $<0.05$ & -0.10 & 0.6 \\
\hline $\begin{array}{l}\text { Septal } \\
\text { thickness }\end{array}$ & 0.28 & $<0.05$ & 0.31 & 0.05 \\
\hline $\begin{array}{l}\text { Presence of } \\
\text { LGE }\end{array}$ & -0.16 & 0.3 & & \\
\hline $\begin{array}{l}\text { Quantity of } \\
\text { LGE }\end{array}$ & 0.16 & 0.3 & & \\
\hline $\begin{array}{l}\text { Post-contrast } \\
\text { myocardial } \\
\mathrm{T}_{1} \text { time }\end{array}$ & -0.48 & $<0.001$ & -0.34 & 0.02 \\
\hline \multicolumn{5}{|c|}{ Echocardiography parameters } \\
\hline $\begin{array}{l}\text { Left atrial } \\
\text { volume } \\
\text { indexed }\end{array}$ & 0.38 & $<0.01$ & 0.24 & 0.06 \\
\hline $\begin{array}{l}\text { Resting LVOT } \\
\text { gradient }\end{array}$ & 0.46 & $<0.001$ & 0.29 & 0.03 \\
\hline
\end{tabular}

NYHA indicates New York Heart Association; LVEDV, left ventricular enddiastolic volume; LVEF, left ventricular ejection fraction; LGE, late gadolinium enhancement; and LVOT, left ventricular outflow tract.

\section{Discussion}

Using a previously histologically-validated post-contrast $\mathrm{T}_{1}$ mapping CMR technique [17], we demonstrated that HCM patients have significantly shorter post-contrast myocardial $\mathrm{T}_{1}$ times compared to healthy controls, consistent with the presence of diffuse interstitial myocardial fibrosis. Furthermore, the amount of this fibrosis correlated with echocardiographic measures of LV filling pressure, suggesting a mechanistic link between diffuse fibrosis and abnormal diastolic function in HCM.

Previously, cardiac biopsy was the only means of comprehensively evaluating both regional and diffuse patterns of myocardial fibrosis. However, invasive endomyocardial biopsy is associated with significant procedural risks and the ability to non-invasively image diffuse fibrosis in patients with HCM would be a significant advance. Additionally, biopsies obtained via this technique are mainly derived from the right ventricular portion of the interventricular septum and may not accurately reflect fibrotic processes occurring in either the hypertrophied LV septum or other ventricular segments.

Research utilizing CMR to assess myocardial fibrosis in typical cohorts of HCM patients has previously only identified regional patterns of fibrosis with LGE sequences [11-14,24]. This focus on LGE in HCM has led to improved accuracy in the diagnosis of this condition in patients with unexplained myocardial hypertrophy and may enhance risk stratification for sudden death [25]. Not all patients with HCM, however, exhibit LGE. Our study detected diffuse myocardial fibrosis in HCM patients both with and without LGE and there was no association observed between the amount of LGE and post-contrast myocardial $\mathrm{T}_{1}$ time. Additionally, post-contrast $\mathrm{T}_{1}$ times of hypertrophied and non-hypertrophied myocardium did not differ, reaffirming the histologically-proven [7] diffuse nature of fibrosis in HCM. In contrast, the presence of LGE correlates with segmental LV wall thickness $[15,26]$. These findings imply that diffuse and regional myocardial fibrosis in HCM are distinct entities. The relative quantities of these two types of fibrosis varied markedly between HCM patients in our study and may partially account for the range of clinical manifestations in this heterogeneous disease. There was a significant overlap in post-contrast myocardial $\mathrm{T}_{1}$ times between $\mathrm{HCM}$ patients and control subjects. Possible explanations for this include; variability in the fibrotic content in the myocardium of healthy controls (including age-related changes); subclinical myocardial disease; and the aforementioned heterogeneity of the extent of diffuse myocardial fibrosis in HCM.

The relationship between reduced post-contrast myocardial $\mathrm{T}_{1}$ times and diastolic dysfunction has been described in a group of patients with clinical heart failure [17]. Recently, in a cohort of heart failure patients 
with preserved systolic function, a correlation was noted between the amount of collagen type 1 found on endomyocardial biopsy and echocardiographic indices of diastolic dysfunction [27]. Utilizing early mitral inflow to early diastolic mitral annular velocity (E/e') as a noninvasive measure of increased LV filling pressure [28-31], our study suggests a mechanistic link between higher LV filling pressures in HCM patients and diffuse myocardial fibrosis. Interestingly, we did not observe a significant correlation between the amount of regional myocardial fibrosis, as detected by LGE, and estimated LV filling pressure.

Animal studies have demonstrated the anti-fibrotic effects of medications that inhibit the angiotensin II system [32]. Therefore, the ability to non-invasively evaluate diffuse fibrosis in HCM is likely to enhance our understanding of pathogenesis and disease progression and may enable therapeutic trials of potential antifibrotic agents. Furthermore, as it is uncertain as to when the active pro-fibrotic state in the myocardium of patients with HCM occurs, serial imaging using $T_{1}$ mapping techniques over a patient's lifetime may glean crucial information about the timing of this process.

\section{Study limitations}

Our research has several limitations. Despite including consecutive patients with asymmetric HCM referred to our CMR centre, no patient experienced class III or IV NYHA symptoms. Further studies involving patients with more severe symptoms, whether due to intracavitary obstruction and/or restrictive physiology, would be required to demonstrate whether patients with a greater disease burden might have even lower postcontrast myocardial $\mathrm{T}_{1}$ times. Also, variations in the timing of image acquisition after contrast administration as well as heart rate, hematocrit and renal function have been proposed as potential confounders to the interpretation of post-contrast $T_{1}$ times [8]. Various $T_{1}$ mapping techniques have been designed to attempt to address these issues, including an approach that utilized a continuous infusion of contrast to achieve equilibrium [20]. A $T_{1}$ mapping technique to calculate the extracellular volume (ECV) of the myocardium has also been described [33]. Currently, no consensus exists on which is the most accurate CMR $\mathrm{T}_{1}$ mapping method, with a number of differing techniques demonstrating significant correlations between post-contrast myocardial $T_{1}$ time and histologically-quantified fibrosis $[17,20]$. We observed no significant differences in baseline values for these putative confounding factors between our study groups and, after statistical analysis, could not identify any significant correlations with any of these factors and post-contrast myocardial $\mathrm{T}_{1}$ times. In addition, postcontrast blood pool $\mathrm{T}_{1}$ times were similar in both groups, strongly suggesting that the lower myocardial $\mathrm{T}_{1}$ times in HCM patients compared to controls were not due to differences in contrast medium kinetics. Importantly, numerous studies have utilized a similar $T_{1}$ mapping technique to that used in our study, and have demonstrated shortened myocardial post-contrast $T_{1}$ times in humans with a wide range of conditions known to be associated with diffuse myocardial fibrosis, including systolic heart failure [17], the diabetic heart [18,34], chronic valvular heart disease [35] and remote myocardial remodelling post-myocardial infarction [36]. Finally, myocardial edema, identified by CMR, has been described in some patients with HCM and may be due to acute ischaemia [37]. Myocardial edema can affect $T_{1}$ times [38], however, while its presence was not directly assessed in this study, there was no clinical evidence of recent acute myocardial pathology in any study patient.

\section{Conclusions}

Using CMR post-contrast $T_{1}$ mapping, this study has demonstrated that patients with HCM have reduced post-contrast myocardial $\mathrm{T}_{1}$ times, consistent with the presence of diffuse interstitial fibrosis. Furthermore, the independent association of post-contrast myocardial $\mathrm{T}_{1}$ time with estimated LV filling pressure $\left(E / e^{\prime}\right)$ suggests a mechanistic link between altered myocardial composition and function. The non-invasive detection of diffuse fibrosis, in combination with standard LGE sequences to identify dense regional fibrosis, now allows a comprehensive evaluation of patterns of fibrosis in this condition. Further research utilizing this technique may enhance our understanding of the relationships between HCM genetic mutations, abnormal myocardial structure and function, and risk stratification and may facilitate the future development of disease-modifying therapies.

\section{Abbreviations}

HCM: Hypertrophic cardiomyopathy; LV: Left ventricle/ventricular; LVOT: Left ventricular outflow tract; CMR: Cardiovascular magnetic resonance; LGE: Late gadolinium enhancement; RV: Right ventricle/ventricular; ASH: Asymmetric septal hypertrophy; eGFR: Estimated glomerular filtration rate; SSFP: Steadystate free precession; ROI: Region of interest; BMI: Body mass index; NYHA: New York Heart Association; BSA: Body surface area; TDI: Tissue Doppler imaging.

\section{Competing interests}

The authors declare that they have no competing interests.

\section{Authors' contributions}

AHE conceived of the study, participated in its design and co-ordination, analyzed echocardiogram and CMR results, and drafted the manuscript. LMI developed the $T_{1}$ mapping technique, completed the ethics submission and analyzed CMR results. LL recruited control patients and analyzed CMR results. JLH analyzed CMR results and assisted in statistical analysis. DMK participated in the study's design and drafted the manuscript. AJT developed the $T_{1}$ mapping technique, participated in the study's design and co-ordination, and drafted the manuscript. All authors read and approved the final manuscript. 
Received: 11 July 2012 Accepted: 17 October 2012

Published: 29 October 2012

\section{References}

1. Maron BJ, McKenna WJ, Danielson GK, Kappenberger LJ, Kuhn HJ, Seidman CE, Shah PM, Spirito P, Ten Cate FJ, Wigle ED, Spencer WH 3rd: American College of Cardiology/European Society of Cardiology clinical expert consensus document on hypertrophic cardiomyopathy. A report of the American College of Cardiology Foundation Task Force on Clinical Expert Consensus Documents and the European Society of Cardiology Committee for Practice Guidelines. J Am Coll Cardiol 2003, 42:1687-1713

2. Maron BJ: Hypertrophic cardiomyopathy: a systematic review. JAMA 2002, 287:1308-1320.

3. Marian AJ, Roberts R: The molecular genetic basis for hypertrophic cardiomyopathy. J Mol Cell Cardiol 2001, 33:655-670.

4. Seidman JG, Seidman C: The genetic basis for cardiomyopathy: from mutation identification to mechanistic paradigms. Cell 2001, 104:557-567.

5. Maron BJ, Spirito P, Shen WK, Haas TS, Formisano F, Link MS, Epstein AE, Almquist AK, Daubert JP, Lawrenz T, et al: Implantable cardioverterdefibrillators and prevention of sudden cardiac death in hypertrophic cardiomyopathy. JAMA 2007, 298:405-412.

6. Fifer MA, Vlahakes GJ: Management of symptoms in hypertrophic cardiomyopathy. Circulation 2008, 117:429-439.

7. Varnava AM, Elliott PM, Sharma S, McKenna WJ, Davies MJ: Hypertrophic cardiomyopathy: the interrelation of disarray, fibrosis, and small vessel disease. Heart 2000, 84:476-482.

8. Mewton N, Liu CY, Croisille P, Bluemke D, Lima JA: Assessment of myocardial fibrosis with cardiovascular magnetic resonance. J Am Coll Cardiol 2011, 57:891-903.

9. Kim RJ, Wu E, Rafael A, Chen EL, Parker MA, Simonetti O, Klocke FJ, Bonow $\mathrm{RO}$, Judd RM: The use of contrast-enhanced magnetic resonance imaging to identify reversible myocardial dysfunction. N Engl J Med 2000, 343:1445-1453.

10. Kim RJ, Judd RM: Gadolinium-enhanced magnetic resonance imaging in hypertrophic cardiomyopathy: in vivo imaging of the pathologic substrate for premature cardiac death? J Am Coll Cardiol 2003 41:1568-1572.

11. O'Hanlon R, Grasso A, Roughton M, Moon JC, Clark S, Wage R, Webb J, Kulkarni M, Dawson D, Sulaibeekh L, et al: Prognostic significance of myocardial fibrosis in hypertrophic cardiomyopathy. J Am Coll Cardiol 2010, 56:867-874.

12. Wilson JM, Villareal RP, Hariharan R, Massumi A, Muthupillai R, Flamm SD: Magnetic resonance imaging of myocardial fibrosis in hypertrophic cardiomyopathy. Tex Heart Inst J 2002, 29:176-180.

13. Teraoka K, Hirano M, Ookubo H, Sasaki K, Katsuyama H, Amino M, Abe Y Yamashina A: Delayed contrast enhancement of MRI in hypertrophic cardiomyopathy. Magn Reson Imaging 2004, 22:155-161.

14. Rubinshtein R, Glockner JF, Ommen SR, Araoz PA, Ackerman MJ, Sorajja P, Bos JM, Tajik AJ, Valeti US, Nishimura RA, Gersh BJ: Characteristics and clinical significance of late gadolinium enhancement by contrastenhanced magnetic resonance imaging in patients with hypertrophic cardiomyopathy. Circ Heart Fail 2010, 3:51-58.

15. Choudhury L, Mahrholdt H, Wagner A, Choi KM, Elliott MD, Klocke FJ, Bonow RO, Judd RM, Kim RJ: Myocardial scarring in asymptomatic or mildly symptomatic patients with hypertrophic cardiomyopathy. J Am Coll Cardiol 2002, 40:2156-2164.

16. Noureldin RA, Liu S, Nacif MS, Judge DP, Halushka MK, Abraham TP, Ho C, Bluemke DA: The diagnosis of hypertrophic cardiomyopathy by cardiovascular magnetic resonance. J Cardiovasc Magn Reson 2012, 14:17.

17. Iles L, Pfluger H, Phrommintikul A, Cherayath J, Aksit P, Gupta SN, Kaye DM, Taylor AJ: Evaluation of diffuse myocardial fibrosis in heart failure with cardiac magnetic resonance contrast-enhanced T1 mapping. J Am Coll Cardiol 2008, 52:1574-1580

18. Ng AC, Auger D, Delgado V, van Elderen SG, Bertini M, Siebelink HM, van der Geest RJ, Bonetti C, van der Velde ET, de Roos A, et al: Association between diffuse myocardial fibrosis by cardiac magnetic resonance contrast-enhanced T mapping and subclinical myocardial dysfunction in diabetic patients: a pilot study. Circ Cardiovasc Imaging 2012, 5:51-59.
19. Amano $Y$, Takayama M, Kumita S: Contrast-enhanced myocardial T1weighted scout (Look-Locker) imaging for the detection of myocardial damages in hypertrophic cardiomyopathy. J Magn Reson Imaging 2009, 30:778-784.

20. Flett AS, Hayward MP, Ashworth MT, Hansen MS, Taylor AM, Elliott PM McGregor C, Moon JC: Equilibrium contrast cardiovascular magnetic resonance for the measurement of diffuse myocardial fibrosis: preliminary validation in humans. Circulation 2010, 122:138-144.

21. Taylor AJ, Al-Saadi N, Abdel-Aty H, Schulz-Menger J, Messroghli DR, Friedrich MG: Detection of acutely impaired microvascular reperfusion after infarct angioplasty with magnetic resonance imaging. Circulation 2004, 109:2080-2085.

22. Saranathan M, Rochitte CE, Foo TK: Fast, three-dimensional free-breathing MR imaging of myocardial infarction: a feasibility study. Magn Reson Med 2004, 51:1055-1060.

23. Maron MS: Clinical utility of cardiovascular magnetic resonance in hypertrophic cardiomyopathy. J Cardiovasc Magn Reson 2012, 14:13.

24. Conte MR, Bongioanni S, Chiribiri A, Leuzzi S, Lardone E, Di Donna P, Pizzuti A, Luceri S, Cesarani F, Mabritto B, et al: Late gadolinium enhancement on cardiac magnetic resonance and phenotypic expression in hypertrophic cardiomyopathy. Am Heart J 2011, 161:1073-1077.

25. Green JJ, Berger JS, Kramer CM, Salerno M: Prognostic value of late gadolinium enhancement in clinical outcomes for hypertrophic cardiomyopathy. JACC Cardiovasc Imaging 2012, 5:370-377.

26. Maron MS, Appelbaum E, Harrigan CJ, Buros J, Gibson CM, Hanna C, Lesser JR, Udelson JE, Manning WJ, Maron BJ: Clinical profile and significance of delayed enhancement in hypertrophic cardiomyopathy. Circ Heart Fail 2008, 1:184-191.

27. Kasner M, Westermann D, Lopez B, Gaub R, Escher F, Kuhl U, Schultheiss HP, Tschope C: Diastolic tissue Doppler indexes correlate with the degree of collagen expression and cross-linking in heart failure and normal ejection fraction. J Am Coll Cardiol 2011, 57:977-985.

28. Nagueh SF, Lakkis NM, Middleton KJ, Spencer WH 3rd, Zoghbi WA, Quinones MA: Doppler estimation of left ventricular filling pressures in patients with hypertrophic cardiomyopathy. Circulation 1999, 99:254-261.

29. Geske JB, Sorajia P, Nishimura RA, Ommen SR: Evaluation of left ventricular filling pressures by Doppler echocardiography in patients with hypertrophic cardiomyopathy: correlation with direct left atrial pressure measurement at cardiac catheterization. Circulation 2007, 116:2702-2708.

30. Matsumura Y, Elliott PM, Virdee MS, Sorajja P, Doi Y, McKenna WJ: Left ventricular diastolic function assessed using Doppler tissue imaging in patients with hypertrophic cardiomyopathy: relation to symptoms and exercise capacity. Heart 2002, 87:247-251

31. Nagueh SF, Bierig SM, Budoff MJ, Desai M, Dilsizian V, Eidem B, Goldstein SA, Hung J, Maron MS, Ommen SR, Woo A: American Society of Echocardiography clinical recommendations for multimodality cardiovascular imaging of patients with hypertrophic cardiomyopathy: Endorsed by the American Society of Nuclear Cardiology, Society for Cardiovascular Magnetic Resonance, and Society of Cardiovascular Computed Tomography. J Am Soc Echocardiogr 2011, 24:473-498.

32. Rocha R, Rudolph AE, Frierdich GE, Nachowiak DA, Kekec BK, Blomme EA, McMahon EG, Delyani JA: Aldosterone induces a vascular inflammatory phenotype in the rat heart. Am J Physiol Heart Circ Physiol 2002, 283:H1802-1810

33. Messroghli DR, Nordmeyer S, Dietrich T, Dirsch O, Kaschina E, Savvatis K, OhI D, Klein C, Berger F, Kuehne T: Assessment of diffuse myocardial fibrosis in rats using small-animal Look-Locker inversion recovery $\mathrm{T} 1$ mapping. Circ Cardiovasc Imaging 2011, 4:636-640

34. Jellis C, Wright J, Kennedy D, Sacre J, Jenkins C, Haluska B, Martin J, Fenwick J, Marwick TH: Association of imaging markers of myocardial fibrosis with metabolic and functional disturbances in early diabetic cardiomyopathy. Circ Cardiovasc Imaging 2011, 4:693-702.

35. Sparrow P, Messroghli DR, Reid S, Ridgway JP, Bainbridge G, Sivananthan MU: Myocardial T1 mapping for detection of left ventricular myocardial fibrosis in chronic aortic regurgitation: pilot study. AJR Am J Roentgenol 2006, 187:W630-635

36. Chan W, Duffy SJ, White DA, Gao XM, Du XJ, Ellims AH, Dart AM, Taylor AJ: Acute left ventricular remodeling following myocardial infarction: coupling of regional healing with remote extracellular matrix expansion. JACC Cardiovasc Imaging 2012, 5:884-893. 
37. Abdel-Aty H, Simonetti O, Friedrich MG: T2-weighted cardiovascular magnetic resonance imaging. J Magn Reson Imaging 2007, 26:452-459.

38. Ferreira VM, Piechnik SK, Dall'armellina E, Karamitsos TD, Francis JM, Choudhury RP, Friedrich MG, Robson MD, Neubauer S: Non-contrast T1-mapping detects acute myocardial edema with high diagnostic accuracy: a comparison to T2-weighted cardiovascular magnetic resonance. J Cardiovasc Magn Reson 2012, 14:42.

doi:10.1186/1532-429X-14-76

Cite this article as: Ellims et al: Diffuse myocardial fibrosis in

hypertrophic cardiomyopathy can be identified by cardiovascular

magnetic resonance, and is associated with left ventricular diastolic

dysfunction. Journal of Cardiovascular Magnetic Resonance 2012 14:76.

\section{Submit your next manuscript to BioMed Central and take full advantage of:}

- Convenient online submission

- Thorough peer review

- No space constraints or color figure charges

- Immediate publication on acceptance

- Inclusion in PubMed, CAS, Scopus and Google Scholar

- Research which is freely available for redistribution 